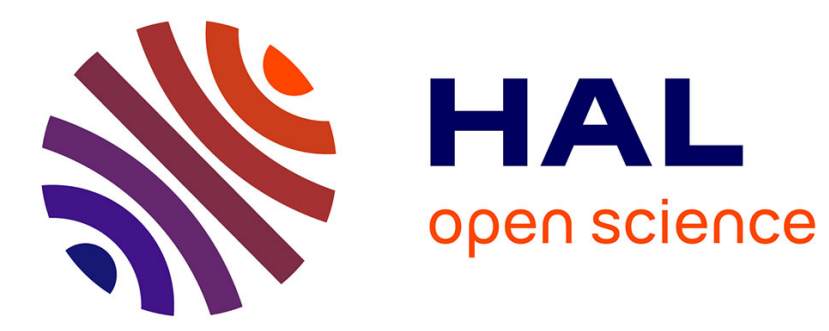

\title{
L'analytique et le synthétique en économie Philippe Mongin
}

\section{To cite this version:}

Philippe Mongin. L'analytique et le synthétique en économie. 2003. hal-00242950

\section{HAL Id: hal-00242950 \\ https://hal.science/hal-00242950}

Preprint submitted on 6 Feb 2008

HAL is a multi-disciplinary open access archive for the deposit and dissemination of scientific research documents, whether they are published or not. The documents may come from teaching and research institutions in France or abroad, or from public or private research centers.
L'archive ouverte pluridisciplinaire HAL, est destinée au dépôt et à la diffusion de documents scientifiques de niveau recherche, publiés ou non, émanant des établissements d'enseignement et de recherche français ou étrangers, des laboratoires publics ou privés. 


\section{ECOLE POLYTECHNIQUE}

CENTRE NATIONAL DE LA RECHERCHE SCIENTIFIQUE

\section{L'analytique et le synthétique en économie}

Philippe Mongin

Avril 2003

Cahier $n^{\circ}$ 2003-005

\section{LABORATOIRE D'ECONOMETRIE}

1rue Descartes F-75005 Paris

(33) 155558215

http://ceco.polytechnique.fr/

mailto:labecox@poly.polytechnique.fr 


\title{
$L^{\prime}$ analytique et le synthétique en économie ${ }^{1}$
}

\author{
Philippe Mongin $^{2}$
}

Avril 2003

Cahier $n^{\circ}$ 2003-005

Résumé: L'article se propose d'appliquer à l'économie la distinction, classique en philosophie des sciences, des propositions analytiques et synthétiques. Après avoir brièvement repris la théorie de cette distinction, il la fait jouer sur deux exemples micro-économiques (les définitions des biens Giffen et des biens substituts) et un exemple macro-économique (celui de l'équation quantitative de la monnaie). Il conclut que la distinction permet de clarifier, généralement sans les heurter, les distinctions spontanées des économistes. Dans le cas des biens substituts, elle permet de révéler au grand jour les difficultés de la définition hicksienne.

\begin{abstract}
The article aims at applying a classic distinction of the philosophy of science, i.e., that between analytical and synthetic propositions. After briefly reconsidering the theory of this distinction, it goes on applying it to two microeconomic examples (the definitions of Giffen goods and substitutes) and one macroeconomic example (the quantity equation in monetary theory). The paper concludes that the distinction makes it possible to clarify the economist's spontaneous distinctions, generally without upsetting them. In the case of substitutes, it highlights the difficulties of Hicks's definition.
\end{abstract}

Mots clés : $\quad$ Analytique, synthétique, Quine, biens Giffen, substituts, équation quantitative, Hicks, Friedman

Key Words : Analytical, synthetic, Quine, Giffen goods, substitutes, quantity equation, Hicks, Friedman

Classification JEL: B41, B21, B22, D11

\footnotetext{
${ }^{1}$ L'auteur remercie de leurs commentaires R. Bradley et J.S. Lenfant.

${ }^{2}$ Laboratoire d'économétrie, Centre National de la Recherche Scientifique et Ecole Polytechnique
} 


\section{L'ANALYTIQUE ET LE SYNTHETIQUE EN ECONOMIE}

"Il semble que les philosophes aient en général tendance à faire des distinctions en nombre insuffisant et non pas en nombre excessif. Ce sont leurs assimilations, et non pas leurs distinctions, qui sont factices" (Grice et Strawson, 1956, p. 144-145).

\section{Introduction}

La distinction de l'analytique et du synthétique est de la plus grande importance pour l'épistémologie économique prise dans son ensemble. En économie plus encore que dans d'autres disciplines au caractère scientifique plus affirmé, un seul et même énoncé peut répondre à des usages théoriques multiples. Il n'est pas toujours facile de séparer les interprétations analytiques et synthétiques de l'énoncé considéré; mais chaque fois qu'on y parvient, on progresse dans la compréhension de la diversité de ses emplois; et, par là même, on appréhende mieux le fonctionnement de la discipline. A ce titre, la distinction est utile et instructive; nous le montrerons en étudiant quelques exemples choisis. La distinction conditionne la manière d'envisager d'autres couples de concepts dont l'épistémologie économique s'occupe en fait plus couramment: l'a priori et l'a posteriori, la syntaxe et la sémantique, la réfutabilité et l'irréfutabilité. Suivant la conception retenue, elle peut déterminer l'articulation d'idées aussi fondamentales que celles de loi et d'explication économiques. Ces apports supplémentaires de la problématique - en particulier la liaison si vivement débattue de l'analytique avec l'a priori - excèdent les limites du présent article.

Comme l'analyticité a fait l'objet de précisions multiples et parfois contradictoires, il nous a paru nécessaire d'en expliciter les définitions utilisables. Nous en avons sélectionné deux: celle - la plus courante aujourd'hui - qui rattache l'analytique aux significations, et celle moins en faveur mais plus facile d'emploi - qui le rattache aux définitions (2). Contre les objections de Quine en 1951 et d'autres auteurs moins radicaux, mais qu'il a influencés, comme Putnam, nous défendrons l'idée que, pour les besoins d'un travail de philosophie des sciences appliquée, comme l'est celui-ci, il est possible d'opérer une distinction satisfaisante de l'analytique et du synthétique (3). Les éclaircissements des deux sections qui viennent ne contiennent pas de grandes nouveautés; ils visent à ranimer une position défendue naguère contre Quine, en particulier par Grice et Strawson, mais qui est restée peu influente chez les 
philosophes, compte tenu de l'avantage pris par la critique quinéenne. Par ces généralités, nous préparons les développements qui constituent le cœur de l'article et sa contribution technique propre. Nous nous servirons de la distinction pour reconsidérer la théorie des biens Giffen (4) et des biens mutuellement substituts (5). En nous attachant ensuite à l'équation quantitative de la monnaie, nous montrerons que la distinction peut servir à raffiner celle que les économistes font passer entre les identités comptables et les relations d'équilibre ou de comportement (6). La dernière section signale plus brièvement d'autres applications, qui se situent non pas au niveau de la théorie, mais au métaniveau du discours méthodologique sur l'économie (7).

Les exemples que nous développons aux sections (4), (5) et (6), proviennent de deux branches, la micro-économie du consommateur et la macro-économie monétaire, qui sont durablement stabilisées, la seconde présentant même une légère coloration d'archaïsme. La plupart des économistes d'aujourd'hui pensent les maîtriser, et cependant, nous verrons que leur démarche gagne en rigueur et en clarté dès qu'on la soumet au tranchant des distinctions épistémologiques. Toute limitée qu'elle soit, la distinction de l'analytique et du synthétique permet non seulement d'élucider, mais même quelquefois de critiquer le travail théorique d'une manière qui puisse finalement importer au praticien lui-même. Nous le montrerons à propos des biens substituts, dont la théorie actuelle, défectueuse aux yeux d'une minorité d'économistes dont nous nous proposons d'étayer les vues, demanderait à être reconstituée sur de nouvelles bases.

\section{L'analytique et le synthétique: définitions}

Pour la philosophie classique, chez Kant notamment, un jugement est synthétique s'il opère une liaison extérieure entre la chose sur laquelle il porte (le "sujet" dans un langage d'école) et les qualités ("prédicats") qu'il lui attribue. On dit que le jugement est analytique lorsque la liaison est interne. ${ }^{1}$ Quant aux propositions, elles seront dites analytiques ou synthétiques selon le jugement dont elles résultent. Une telle manière de présenter la question est datée, parce qu'elle repose sur une théorie du jugement que les modernes regardent avec méfiance elle serait "psychologisante" - et parce qu'elle a partie liée avec une logique formelle désuète -

\footnotetext{
${ }^{1} \mathrm{Cf}$ : "Ou le prédicat B appartient au sujet A comme quelque chose qui est contenu d'une manière cachée dans ce concept $\mathrm{A}$, ou $\mathrm{B}$ est entièrement en dehors du concept $\mathrm{A}$, quoiqu'il soit, à la vérité, en liaison avec lui. Dans le premier cas, je nomme le jugement analytique; dans le second, synthétique" (Kant, Critique de la raison pure, Introduction, 1ère éd., IV). Hintikka (1973) et, plus systématiquement, Proust (1986) ont commenté l'histoire de cette définition.
} 
celle du syllogisme, par opposition aux calculs des propositions et des prédicats. Jusqu'à une date avancée, on a continué de discuter les propositions économiques en s'appuyant sur la théorie du jugement et la syllogistique plutôt que sur les conceptions modernes; il n'était donc pas entièrement déplacé de rappeler la définition des classiques avant de passer à celles dont nous ferons usage. ${ }^{2}$

Les philosophes contemporains placent leur distinction technique au plan des propositions, sans se référer à un acte de jugement antérieur. Suivant la définition la plus courante, elle déclare analytiques les propositions dont la vérité ou la fausseté dépend seulement de la signification des termes figurant dans les énoncés qui expriment ces propositions. "Analytique" s'emploie, par abréviation, pour désigner les propositions analytiques vraies par opposition à toutes les autres, mais la définition permet naturellement qu'il y ait des propositions analytiques fausses. On appelle synthétiques toutes celles qui ne sont pas analytiques: les deux catégories sont prises comme exclusives et exhaustives. Toujours suivant la définition courante, la synthéticité d'une proposition se manifeste aussi de manière positive: sa valeur de vérité dépend, entre autres choses, des faits qu'elle indique. Il est sousentendu que, si les significations ne déterminent pas entièrement la vérité ou la fausseté, celles-ci dépendent de ce qu'est le monde réel. ${ }^{3}$

On peut sans contradiction avec le sens des mots "corps", "tombe", "vide", se représenter des corps tombant avec une vitesse constante plutôt qu'avec une accélération constante, et une pareille conception semble d'ailleurs avoir préexisté à celle de Galilée. Si elle est fausse, c'est uniquement parce qu'elle ne s'accorde pas avec le monde physique qui est le nôtre, et non parce qu'elle serait absurde en elle-même. On opposera donc la loi de Galilée, proposition synthétique dont la physique décide si elle est vraie ou fausse, à celle-ci, qui est analytiquement fausse: les corps ne sont pas étendus. La proposition entre en conflit avec les significations puisque "corps" suppose "étendu". On peut opposer encore la loi de Galilée aux propositions arithmétiques: suivant la thèse qui, sans être universelle, est la plus couramment défendue, celles-ci seraient analytiquement vraies. On ne concevrait pas de contredire " $2+2=$ $4 "$, sauf à changer le sens attribué aux nombres entiers, à l'opération d'addition et à la relation d'égalité.

2 Cet archaïsme se révèle dans le débat convenu sur les jugements de valeur en économie, où il a des effets catastrophiques.

3 Cf: "Un énoncé est analytique quand il est vrai en vertu des significations et indépendamment des faits" (Quine, 1951-1961, p.21). Voir aussi Carnap (1947-1956, p. 222) 
Sensiblement différente de celle qui précède, la définition suivante se rencontre aussi dans la philosophie du $\mathrm{XX}^{\mathrm{e}}$ siècle: une proposition est analytique si l'on peut connaître sa valeur de vérité en utilisant seulement les lois de la logique et les définitions explicites des termes employés. Elle est synthétique dans le cas contraire. ${ }^{4}$ Pour le dire plus précisément, une phrase représente une proposition analytiquement vraie si elle peut s'obtenir à partir, seulement, de phrases représentant des vérités logiques, comme "A=A" ou "p ou non p"; des règles d'inférence coutumières; enfin, de définitions explicites, c'est-à-dire de phrases de la forme: "x est $\mathrm{A}$ si et seulement si...".5 Le commentaire donne à penser que les "lois de la logique" seraient du type ordinaire: c'est la situation elle-même la plus ordinaire en science. Dans les cas exceptionnels où il n'en va pas ainsi, en mécanique quantique par exemple, les "lois" déviantes seront expressément indiquées, et c'est alors par rapport à elles que l'on jugera du caractère analytique ou non des propositions. Frege, qui est à l'origine de la définition que nous considérons maintenant, s'intéressait aux propositions de l'arithmétique, dont il voulait établir, contre Kant, qu'elles étaient analytiques. ${ }^{6}$ On peut se prévaloir de la conception frégéenne bien au-delà de cette application privilégiée. Où que ce soit, elle présente l'avantage d'être simple, voire même opératoire une fois qu'on a décidé des définitions à retenir. Elle débouche en effet sur un algorithme de vérification: une proposition sera dite analytique si elle se ramène à l'égalité "A=A", une fois qu'on a effectué les transformations permises par le calcul logique et par les définitions explicites que l'on a préalablement rajoutées à ses axiomes.

La définition frégéenne inclut automatiquement les vérités logiques - que nous appellerons aussi tautologies - dans l'ensemble des propositions analytiques. Toute proposition qui se ramène à une vérité logique connue sans que l'algorithme fasse intervenir autre chose que la logique elle-même doit être à son tour classée parmi les vérités logiques. La définition courante inclut de même les vérités logiques, mais la raison proposée diffère: celles-ci correspondent au cas particulier où la valeur de vérité se décide en examinant la signification des termes logiques seulement. Ainsi, "Tout corps est un corps" et "Tout corps est étendu ou certains corps ne sont pas étendus" énoncent des tautologies, à la différence de "Tout corps est

\footnotetext{
4 "Si l'on ne rencontre sur (son) chemin que des lois logiques générales et des définitions, on a une vérité analytique..." (Frege, 1884, §3). La philosophie des sciences contemporaine emploie cette définition moins couramment que l'autre, ainsi que le souligne Gillies (1993).

5 Il est courant qu'on s'accorde spontanément sur les définitions en question, de sorte qu'en dépit de leur dénomination, elles n'ont alors pas besoin d'être explicitées.
} 
étendu". Il suffit de connaître le sens des termes logiques "est", "tout", "certains", "ou" pour s'assurer que les deux premières phrases énoncent des vérités, alors qu'il faut connaître aussi le sens de termes non logiques - "corps" et "étendu" - pour élucider la troisième phrase. Dans l'une et l'autre conception, le découpage entre les vérités logiques et les autres propositions analytiques dépend de la logique retenue, mais si l'on s'en tient aux calculs ordinaires, il s'effectue sans difficulté; et de toutes les façons, la distinction interne paraît claire dans le principe. Comprise avec la réserve convenable, elle permet au philosophe des sciences de restreindre son objet, en abandonnant une fois pour toutes au logicien l'étude des tautologies.

Dans Meaning and Necessity, Carnap a proposé une définition de l'analyticité que l'on traite parfois comme distincte, mais qu'il vaut mieux voir comme une formalisation de la définition courante, adaptée aux langages de la logique contemporaine. Sommairement parlant, Carnap construit un de ces langages, L, et définit une formule comme analytique si elle est vraie en fonction, seulement, des règles d'interprétation adoptées pour L. Carnap a fluctué dans sa manière de formaliser les règles d'interprétation, et il a fini par les représenter comme des postulats spéciaux qu'il conviendrait d'ajouter aux axiomes purement logiques du langage. Les détails de sa définition n'importent guère parce qu'elle ne permet ni de se passer des concepts dont on a besoin pour élucider l'analytique, ni de comprendre ces notions mêmes. Au contraire, il faut avoir une idée préalable de ce qu'on appelle "interprétation" si l'on veut énoncer les postulats convenables. Par exemple, c'est parce qu'on possède une idée de ce genre que l'on décidera que "célibataire" s'interprète comme "non marié", la décision faisant que l'on pose le postulat spécial: "Tout célibataire est un homme non marié". Les prémisses ainsi rajoutées aux axiomes logiques n'auront pas exclusivement la forme de définitions explicites; c'est en quoi la construction sémantique penche du côté de la définition courante, et non pas du côté de la définition frégéenne, que Carnap contestait d'ailleurs à d'autres égards encore. 7

La relation des trois principaux concepts d'analyticité pose des questions délicates, mais on peut admettre assez facilement que la définition frégéenne étend la définition classique de l'analytique à certaines propositions que Kant ou Leibniz, prisonniers du schéma "sujetprédicat", n'envisageaient pas comme telles; et nous admettrons de même que la définition

\footnotetext{
${ }^{7}$ La définition frégéenne lui semblait trop proche encore de celles des classiques. Il est douteux que Meaning and Necessity constitue le progrès désiré. La pente de Carnap, exactement opposée à celle de Frege, est qu'il ne marque pas assez la différence entre les vérités logiques et les propositions analytiquement vraies qui ne sont pas des vérités logiques.
} 
courante étend la définition frégéenne, puisque la notion de signification constatée va plus loin que celle de définition explicite. Dans la communication quotidienne, les significations se révèlent autrement que par des définitions de la forme "x est A si et seulement si...". Ce n'est pas la manière, par exemple, dont un dictionnaire les donne à connaître; ce n'est pas non plus en s'appuyant sur des définitions explicites que les sciences procèdent le plus souvent. Il est banal qu'elles fixent des significations pour les termes techniques non pas un à un, mais globalement, tout en reliant ces termes non par de simples implications. L'apport le plus durable de Carnap à la problématique de l'analyticité n'est pas sa théorie des règles d'interprétation, mais le fait qu'il ait su mettre ce point simple en évidence. ${ }^{8}$ Le mode de présentation le plus courant en science, la définition implicite, ne diffère pas par nature de celui du dictionnaire: les termes y sont définis solidairement et, dans une certaine mesure, circulairement. La différence avec le dictionnaire est plutôt de degré: comme on l'a souvent remarqué, celui-ci forme une chaîne circulaire pratiquement unique, alors que les sciences introduisent leurs termes par grappes volontairement restreintes.

Malgré tant d'efforts accomplis pour la clarifier, tous les philosophes contemporains ne sont pas convaincus que la distinction de l'analytique et du synthétique ait vraiment lieu d'être. Nombre d'entre eux rejettent même ce que nous avons désigné - trop vite sans doute - comme la conception courante de l'analytique. Pour ceux-là, la distinction serait non seulement imprécise, comme le sont tant d'autres distinctions philosophiques, mais irrémédiablement mal fondée. Ils répercutent la thèse illustre de Quine dans From a Logical Point of View, tellement influente aujourd'hui qu'on doit pratiquement renoncer à parler d'analyticité si l'on ne réussit pas à se prémunir contre elle. La section qui suit, strictement générale encore, s'efforce de parer aux objections importantes qu'elle soulève. ${ }^{9}$

\section{Pour défendre la distinction de l'analytique et du synthétique}

La critique de Quine porte non pas sur les langues formelles, qu'il met à part, mais sur la langue ordinaire, à laquelle il emprunte son exemple rebattu par la suite: faut-il ou non lire une proposition analytique ou non dans "les célibataires ne sont pas mariés"? Un premier jeu d'arguments vise à établir, en substance, que l'on ne parvient pas à comprendre la notion

\footnotetext{
8 Non seulement dans Meaning and Necessity, mais surtout dans "Testability and Meaning" (1936-37), où il en tire des conséquences utiles pour la philosophie des sciences.

${ }^{9}$ L'évolution de Quine au-delà de cet article célèbre ne nous retiendra pas ici; nous renvoyons à Gochet (1978) et Haack (1978).
} 
d'analytique si l'on ne comprend pas d'abord celle de signification, qui renvoie à son tour à d'autres notions du même registre, comme celles de synonymie, de substitution valide d'une expression à une autre et, finalement, de définition. Or ces différentes notions formeraient un cercle avec la notion initiale et, par là même, ne permettraient pas de l'élucider convenablement. Comme certains l'ont aussitôt remarqué, il est possible de rompre le cercle prétendu si l'on se satisfait de comprendre les notions manquantes par des exemples: le modèle de la définition que l'on adopte est alors l'ostension - on montre les cas où, selon toute apparence, le concept s'applique correctement. De plus et surtout, la circularité, même si elle est bien réelle, ne peut constituer en elle-même une objection. Plus haut, nous rappelions que les définitions des dictionnaires sont toutes peu ou prou comprises dans des cercles; or il n'y a pas de doute qu'elles facilitent la compréhension des termes qu'elles introduisent ainsi. Il semblerait que, sans le reconnaître, Quine ait voulu confronter l'analyticité à un objectif d'élucidation fortement spécifique. Grice et Strawson (1956) affirment que ses objections tomberaient s'il ne s'imposait pas de trouver une définition explicite pour chacun des concepts qu'il aborde tour à tour. Quelles que soient précisément les exigences métathéoriques de Quine, il nous paraît certain que, dans son texte fameux, il en demande trop: son exigence de clarté passe alors les moyens dont on dispose couramment pour expliquer les notions philosophiques; elle est hors de propos. ${ }^{10}$

Une autre partie de la critique de Quine procède des considérations suivantes, qui sembleront plus solidement fondées. Pour se servir pratiquement de la distinction de l'analytique et du synthétique, il faut pouvoir accéder aux significations. Or il existe un moyen connu pour connaître les significations visées par les phrases: on se reporte à la manière de vérifier ou d'infirmer ce qu'elles disent. Ce critère se transpose immédiatement à la synonymie: on reconnaîtra deux phrases comme synonymes si elles sont équivalentes du point de vue des moyens de vérification ou d'infirmation. ${ }^{11}$ On ne demandera pas que les deux phrases aient donné lieu à des vérifications ou des infirmations effectives pour qu'on puisse les déclarer ou non synonymes: il suffit de considérer les vérifications et les infirmations concevables. On obtient finalement un procédé de reconnaissance de l'analytique: on dira qu'une proposition est analytiquement vraie si la phrase qui l'exprime est synonyme, au sens qui vient d'être dit, de la formule d'une vérité logique. Quine conteste le critère initial de repérage des significations, dont tout le reste dépend. Grice et Strawson voulaient montrer que ses

10 Ces objections à Quine se retrouvent dans certains manuels contemporains, comme celui de Grayling (1997).

11 Quine le dit ainsi: "Deux énoncés sont synonymes si, et seulement si, ils sont les mêmes relativement à la méthode de confirmation ou d'infirmation" (Quine, 1951-1961, p. 37). 
objections n'étaient pas probantes tout en défendant le critère lui-même. Nous ne les suivons pas et, dans le cas d'espèce, renchérissons bien volontiers sur la position quinéenne. Certaines phrases visent des faits du monde qu'on ne voit pas comment apprécier empiriquement; or le critère ne permet pas de les traiter, et si on le durcit au point d'en faire une définition, il faudrait conclure qu'elles sont dénuées de signification, ce qui serait un diagnostic singulier et, dans certains cas, proprement aberrant. En outre, le critère vérificationniste est, dans la pratique, ambigu et difficile à appliquer. Ces observations critiques sont aujourd'hui très bien comprises. Mais on peut en faire l'hommage à Quine, qui a été l'un des premiers à les faire valoir, et, pour autant, s'étonner qu'il rejette la distinction de l'analytique et du synthétique en général au motif qu'une conception particulière de la signification qui servirait à la fonder s'avère inadéquate. La définition frégéenne de l'analytique, par exemple, ne présupposait pas l'idée précédente des significations et de la synonymie. Il faut conclure que la partie de la critique quinéenne dont nous venons de traiter n'a que la force limitée d'une argumentation ad hominem. Elle porte seulement contre ceux qui, pendant une phase d'ailleurs circonscrite du positivisme logique, revendiquèrent la doctrine qu'il est convenu d'appeler vérificationnisme. Ce n'est pas rabaisser complètement Quine que d'affirmer que ses arguments paraissent aujourd'hui datés; on ne fait que circonscrire mieux sa contribution. ${ }^{12}$

Quitte à négliger bien des subtilités intermédiaires, nous conclurons que la critique de Quine en 1951 n'aboutit pas. Mais l'esprit qui l'inspirait, sinon la lettre de ses objections, importe tout de même, et pour l'apprécier, il faut entrer, si peu que ce soit, dans la thèse positive que From a Logical Point of View développait simultanément: celle du holisme épistémologique. Afin de mettre en défaut l'idée vérificationniste de la signification, Quine soulignait avec force que, dans la vie ordinaire comme en science, il n'est ni courant, ni même possible de se fixer sur des proposition isolées. Pour décider si une phrase indique une vérité ou une fausseté, il faut impliquer dans l'examen d'autres phrases qui forment avec elle un tout. Ainsi que Duhem (1906) l'avait fort bien expliqué pour ce qui concerne la physique, on ne peut décider de la vérité ou de la fausseté d'une conclusion expérimentale sans supposer réalisées les différentes conditions de l'expérience, comme la correction des instruments de mesure et l'absence d'effet perturbateur. On peut transposer les affirmations de Duhem et de Quine de la vérité à la signification même si l'on n'est pas vérificationniste. Quelle que soit la manière dont on comprenne celle-ci, par la vérification ou par tout autre moyen, il paraît clair que l'on ne peut pas la déterminer en examinant seulement des phrases isolées. C'est l'ensemble du

12 Katz (1990) développe une critique parallèle à celle-ci lorsqu'il rattache la réfutation de Quine à un état dépassé de la linguistique, celui du béhaviorisme défendu par Bloomfield. 
discours, et non pas la phrase ou le mot, qui représente l'unité convenable pour dégager les interprétations. Pour être banale, la thèse frappe comme correcte et importante. Nous l'appliquerons en prenant comme un tout la théorie du consommateur lorsque viendra le moment de dissiper ses obscurités sémantiques.

A cette thèse de globalité, nous pouvons en adjoindre une autre, également présente chez Quine et tout aussi peu surprenante, que nous pouvons dire d'intentionnalité: l'interprétation demande d'appréhender les objectifs de l'auteur, et le même discours, en particulier scientifique, peut appeler des décisions variables suivant les intentions, elles-mêmes variables, que l'on peut mettre derrière ses paroles. S'agissant des mots, nous le verrons à propos de l'expression "bien Giffen": elle veut dire tantôt une chose, tantôt une autre, dans l'esprit des économistes qui l'emploient. S'agissant des phrases, nous verrons comment fluctue la vérité de ce qu'affirme celle-ci: "la demande d'un bien décrô̂t avec son prix sauf s'il s'agit d'un bien Giffen". Les deux thèses que nous venons de formuler sont distinctes, mais il apparaît qu'elles sont étroitement liées entre elles. C'est en prêtant attention aux phrases toutes ensemble, et non pas une à une, que, pour un discours quelconque, on peut espérer saisir les objectifs de son auteur. Le contexte interne de la phrase - tel qu'il est fourni par l'œuvre et, dans notre cas, par le corpus de la théorie économique - ne livre sans doute pas toujours et en totalité ce que le premier voudrait établir à propos du second. Il peut être nécessaire d'examiner le contexte externe, qui est celui de l'énonciation elle-même: les circonstances de temps et de lieu où la phrase a été prononcée, imprimée, diffusée. Il nous semble cependant que le choix et la bonne intelligence des informations supplémentaires, qui sont fuyantes et complexes à mobiliser, dépendront de la prise que l'étude du discours permet d'avoir sur les intentions latentes. En bref, le contexte interne prime sur le contexte externe quand il faut établir les intentions directrices. Ce n'est pas le lieu d'argumenter en faveur d'un tel principe contre les objections répétées de l'histoire et de la sociologie des sciences. Dans cet article, nous le tiendrons simplement pour acquis. Les interprétations qu'il propose du travail des économistes en feront sentir l'intérêt général: le lecteur constatera qu'elles permettent de rendre compte des difficultés sémantiques de la théorie sans qu'il soit nécessaire de sortir jamais du contexte interne.

On peut reformuler certaines conséquences du holisme méthodologique en explicitant la distinction, aujourd'hui courante, mais que Quine lui-même ne pratiquait pas en 1951, entre, d'une part, les énoncés ou les phrases, et d'autre part, les propositions. Les premiers sont des assemblages de signes dans un langage donné, qui peut être la langue ordinaire aussi bien 
qu'un langage artificiel. Pour désigner l'ensemble de cette catégorie, nous préférerons désormais le mot "énoncé" à celui de "phrase" parce qu'il s'applique mieux aux langues artificielles et qu'il est impératif de réintégrer celles-ci dans la discussion. Les secondes sont des entités au statut mal défini, et qui font l'objet de théorisations diverses, voire conflictuelles, mais dont le rôle fonctionnel est généralement clair: on les introduit à côté des énoncés pour qu'il soit possible d'invoquer à leur propos la signification et les autres notions du même registre. C'est la proposition qu'exprime l'énoncé, et non pas l'énoncé lui-même, qui est responsable des significations ainsi que des rapports de signification, comme la synonymie, et c'est elle qui porte les valeurs de vérité. ${ }^{13}$ Dans cette terminologie, le holisme épistémologique a pour conséquence générale que seul le contexte permet d'apercevoir ce qu'est la proposition associée à un énoncé donné. Il s'agit sans doute à nouveau d'une banalité, mais elle forme une conclusion solide, et nous en ferons l'application dans tout ce qui suit. Ainsi réduit à trois affirmations essentielles - globalité, intentionnalité, dépendance contextuelle des propositions - le holisme méthodologique ne paraît finalement guère menaçant pour la distinction de l'analytique et du synthétique.

La valeur de vérité des propositions et la signification des termes dans la proposition déterminent le diagnostic d'analyticité - la définition frégéenne privilégiant la vérité, la définition plus générale insistant sur la signification. Comme ces deux notions s'appliquent aux propositions, il en ira de même pour celle d'analyticité, et la thèse de contextualité se transpose ainsi: suivant le cas, un même énoncé peut renvoyer soit à une proposition analytique, soit à une proposition synthétique. La difficulté à porter le diagnostic peut être considérable, mais le point qu'il faut souligner est qu'elle est entièrement comprise dans la difficulté première à identifier la proposition: l'analyticité n'ajoute pas d'obscurité supplémentaire. Parmi les catégories de la sémantique, celle-ci n'appelle aucune réserve ou précaution spéciale, contrairement à ce que Quine n'a cessé de prétendre. ${ }^{14}$

Il reste une dernière étape à franchir pour défendre la distinction. Le holisme méthodologique en quelque sorte banalisé dont nous venons de décrire la position ne comporte-t-il pas le risque de diluer excessivement les concepts sémantiques? La facilité apparente des thèses précédentes ne dissimulerait-elle pas leur pouvoir dissolvant? S'agissant de l'analytique et du

\footnotetext{
13 Certains pratiquent une division plus raffinée entre les phrases (sentences), vues comme assemblages de signes, les énoncés (statements), qui font proprement l'objet de l'assertion, et les propositions (propositions), conçues comme les significations données aux phrases. Cette tripartition n'est pas indispensable ici.

14 Voir (1951-1961, p. 138 et passim).
} 
synthétique, de nombreux philosophes ont conclu, et concluraient encore de la discussion précédente, que la distinction de ne se heurte pas tant à une impossibilité de principe qu'à une difficulté de mise en œuvre. L'objection se rencontre souvent; il faut essayer maintenant d'y parer. ${ }^{15}$

On a souvent objecté que certaines propositions scientifiquement importantes - les principes de conservation en physique, par exemple - opposaient de sérieuses résistances au diagnostic. A cela, on peut répondre que la distinction de l'analytique et du synthétique affleure immédiatement dans d'autres applications des sciences qu'il serait absurde de négliger, et que les moyens de trancher ne manquent même pas toujours dans les cas équivoques. Une chose est d'admettre que l'utilisateur de la distinction doive prendre des décisions sémantiques, une autre est de prétendre que ses décisions tomberaient dans l'arbitraire. C'est un glissement pur et simple qui permet de passer d'une idée à l'autre, comme si la nécessité de faire un choix impliquait qu'il dût être sans raison; le contexte peut très bien jouer de manière systématique sur les interprétations.

Sans prétendre l'argumenter jusqu'au bout, nous admettrons cette quatrième thèse, qui est extérieure au holisme méthodologique: dans une discipline constituée, les choix d'interprétation découlent du contexte, interne et, s'il le faut, externe, suivant des règles qui sont relativement fixes et connaissables. Les contextes propres à ces disciplines sont très souvent stéréotypés. Il en va particulièrement ainsi quand le discours du savant répond à l'une des activités scientifiques recensées, comme la démonstration logico-mathématique, l'explication, l'unification théorique; il en va de même encore quand il répond à des fonctions rhétoriques générales, comme la production surdéterminée de preuves, la transposition analogique de résultats antérieurs, la contestation d'un adversaire nommément désigné. Or chaque stéréotype appelle spontanément son genre de décisions sémantiques et, éventuellement, pragmatiques. Lorsque, par exemple, un énoncé micro-économique obéit à une intention explicative affirmée, l'interprétation synthétique s'impose; il en irait différemment si le même énoncé figurait dans une élucidation d'économie mathématique. La démarcation du corpus pertinent soulève une difficulté, mais nous admettrons - à nouveau sans développer l'argumentation - que les découpages théoriques préexistants constituent des points d'arrêt naturels pour l'interprète. Ainsi, la théorie micro-économique des biens

15 Les arguments de Putnam (1975, ch. 2), souvent cités, ne mettent pas en cause l'existence de la distinction, mais sa pertinence et son applicabilité. Putnam n'insiste pas tant sur la diversité des contextes, comme nous le faisons, que sur la pluralité des critères de signification à prendre en compte. 
substituts définit un premier niveau convenable pour discuter le caractère analytique ou non des propositions portant sur ces biens; il est quelquefois préférable de monter à un second niveau, celui de la théorie du consommateur, voire à un troisième, celui de la micro-économie tout entière. On ne peut pas dire à l'avance où il faut s'arrêter, mais on sait du moins que le niveau pertinent relève d'une échelle préassignée.

En mécanique, l'équation $F=m g$ constitue le plus souvent une définition explicite de la masse. Toutefois, il arrive que les physiciens choisissent de définir et de mesurer la masse indépendamment de la force et de l'accélération (ils peuvent se servir d'un ressort de Hooke). L'équation $F=m g$ énonce alors une loi de la physique newtonienne. La différence des deux usages est reconnue depuis longtemps par les physiciens, quoique, naturellement, ils n'emploient pas la terminologie philosophique pour la décrire. Avec cet exemple, on se trouve dans le cas le plus favorable, celui où la discipline elle-même fixe l'interprétation. Des complications surgissent quand on passe aux énoncés dérivés à l'aide de l'équation $F=m g$. Il ne suffit plus alors de repérer les stéréotypes agissants sur l'énoncé particulier; il faut examiner la chaîne implicative entière, avec toutes ses prémisses rajoutées. Des difficultés plus grandes encore se présentent lorsqu'il faut reconstituer une sémantique en l'absence de toute définition explicite: on quitte alors le terrain de la conception frégéenne, sur lequel il est préférable de se tenir quand cela est possible. Putnam (1975) cite le principe d'Einstein suivant lequel les lois de la physique doivent être invariantes par rapport aux transformations de Lorentz. On ne peut évidemment s'appuyer sur une définition, explicite ou non, de ce que sont les lois de la physique pour décider si le principe est analytique. Il apparaît que des cas pareils ne puissent tomber sous la distinction qu'au prix d'un diktat sans justification.

De cette revue expéditive, il ressort que la philosophie des sciences fait face à une gradation continue de diagnostics. L'exemple de Putnam figure vers l'une des extrémités de l'échelle, et la difficulté de mise en œuvre est bien moins générale qu'il ne veut le donner à penser. Il se trouve que nous n'avons jamais rencontré, dans notre enquête, de cas aussi gênant que le principe d'Einstein. S'agissant de l'équation quantitative, les distinctions tracées par les économistes peuvent être cohérentes avec celle de l'analytique et du synthétique, à condition de la prendre dans la version tantôt frégéenne, tantôt généralisée. Quant à la théorie des biens mutuellement substituts, elle répond pratiquement au modèle de $F=m g$. Elle ne laisse pas de doute sur les définitions explicites qu'il faut retenir; c'est un de ces cas où le point de vue frégéen suffit à un examen complet. Il reste en revanche à étudier le statut analytique ou non des conséquences lointaines, et à ce point de complexité, le diagnostic peut devenir 
surprenant. Nous commençons par un cas d'une espèce encore différente: il se place en quelque sorte non pas au-delà, mais en deçà, du modèle fourni par $F=m g$. Les économistes disposent de plusieurs définitions explicites pour la notion de bien Giffen comme les physiciens pour celle de masse; mais contrairement à ceux-ci, ils ne choisissent pas toujours avec la netteté convenable entre les différentes possibilités. La section montrera qu'il est possible de le faire à leur place tout en respectant les indications du contexte interne, c'est-àdire d'une manière qu'ils pourraient finalement eux-mêmes accepter.

\section{Les biens Giffen: entre l'analytique et le synthétique}

Les biens Giffen entrent dans la théorie micro-économique grâce à Marshall, qui rapporte dans ses Principles of Economics (1890-1920, p.109-110) l'observation suivante, due à Sir Robert Giffen: dans certaines familles pauvres, lorsque le prix du pain augmente, sa consommation augmente aux dépens des autres biens. Il s'agit d'une violation saisissante de la loi de la demande, la première d'une longue liste d'exceptions. La catégorie des biens Giffen recouvre d'autres cas identifiés vers la même époque: lorsque le prix de la pomme de terre augmentait, sa consommation, chez les Irlandais les plus misérables, avait tendance à croître aux dépens, cette fois-ci, du pain. Dans tous les cas, le bien est un produit de première nécessité, consommé par des familles pauvres qui lui consacrent une part importante de leur revenu, comportant un ou plusieurs substituts naturels. Même si Marshall en ébauche déjà l'explication, le cas Giffen se présente historiquement comme une simple découverte empirique. Dans Value and Capital, de Hicks (1939), qui inaugure la manière contemporaine de traiter les biens Giffen, l'exception va s'apprécier à la lumière de considérations théoriques précises, en l'occurrence, de la décomposition que les manuels reprennent aujourd'hui: une variation de la demande en fonction du prix se réexprime comme la somme de deux effets distincts et parfois opposés, l'effet de substitution et l'effet de revenu.

Mathématiquement, nous noterons par $x=\left(x_{1}, \ldots, x_{l}\right) \in R_{+}^{l}$ les vecteurs de demande des $l$ biens, par $p=\left(p_{1}, \ldots, p_{l}\right) \in R_{+^{*}}^{l}$ le vecteur des prix correspondants, et par $I$ le budget du consommateur. En supposant que celui-ci maximise une fonction d'utilité $u(x)$ sous la contrainte de budget $p \cdot x=I$, la théorie dérive les demandes de chaque bien $j$ comme des fonctions de $p$ et de $I, x_{j}(p, I)$, et elle établit les équations suivantes, dites de Slutsky. ${ }^{16}$ Celles-ci sont valables pour deux biens quelconques $j$ et $k$, et pour tous les couples de prix et

16 Par hypothèse, la fonction $u$ est ordinale, c'est-à-dire définie à une transformation monotone croissante près, et satisfait les conditions habituelles pour l'existence d'un maximum. 
de revenus $(p, I)$ :

$(*) \frac{\partial x_{j}(p, I)}{\partial p_{k}}=\frac{\partial h_{j}(p, \bar{u}(p, I))}{\partial p_{k}}-\frac{\partial x_{j}(p, I)}{\partial I} \cdot x_{k}(p, I)$

Dans ces équations, $\bar{u}(p, I)$ désigne la valeur de l'utilité $u$ obtenue, par maximisation, pour le couple $(p, I)$ considéré - ce qu'on nomme l'utilité indirecte du consommateur en $(p, I))$ - et $h_{j}(p, \bar{u})$ représente la demande du bien $j$ exprimée comme une fonction des prix et du niveau atteint par la fonction d'utilité - ce qu'on nomme la demande compensée en $(p, \bar{u}))$. Pour bien la distinguer de $h_{j}(p, \bar{u})$, on désigne aussi la notion initiale de demande $x_{j}(p, I)$ comme la demande brute.

Grâce aux équations de Slutsky, on peut décomposer l'effet $\Delta x_{j}$ d'une petite variation $\Delta p_{k}$ du prix de $k$, toutes les autres variables restant fixées:

$\Delta x_{j} \approx \frac{\partial x_{j}(p, I)}{\partial p_{k}} \cdot \Delta p_{k}=\frac{\partial h_{j}(p, \bar{u}(p, I))}{\partial p_{k}} \cdot \Delta p_{k}-\frac{\partial x_{j}(p, I)}{\partial I} \cdot x_{k} \cdot \Delta p_{k}$

D'après la dernière équation, la variation induite par celle du prix de $k$ sur la demande du bien $j$ se présente comme la somme de deux variations élémentaires: la variation qui se produirait si le consommateur demeurait au niveau d'utilité précédent (effet de substitution) et celle qui provient de la modification du revenu que provoque implicitement le changement de prix (effet de revenu).

Dans le cas où $j \neq k$, les équations $(*)$ donnent un moyen d'examiner les relations de substitution ou autres qui se présentent entre des biens différents; tel est l'objet de la théorie discutée à la section suivante. $\mathrm{Si}$, en revanche, on prend $j=k$, les équations permettent d'étudier la demande de chaque bien $j$ en fonction de son prix $p_{j}$, toutes les autres variables restant fixées. Le problème des biens Giffen doit être examiné dans ce cas. La théorie le résout en considérant les poids respectifs, dans $\Delta x_{j}$, des deux effets de substitution et de revenu propres, c'est-à-dire provoqués par $\Delta p_{j}$. Voici, à cet égard, le dernier rappel nécessaire: la théorie parvient à déterminer le signe de l'effet de substitution propre, mais non pas celui de l'effet de revenu propre. On démontre que si le consommateur maximise son utilité $u(x)$ sous la contrainte budgétaire $p x=I$, alors:

$\frac{\partial h_{j}(p, \bar{u}(p, I))}{\partial p_{k}}<0$

tandis que: 
$-\frac{\partial x_{j}(p, I)}{\partial I} \cdot x_{k}$

peut-être de signe quelconque. ${ }^{17}$

Compte tenu de ces résultats qui leur sont bien connus, de nombreux économistes croient bon d'adopter la définition suivante, elle-même théorique et abstraite, d'un bien Giffen: toute espèce de bien j tel que l'effet de revenu qui lui est associé excède l'effet de substitution. 18 D'autres demeurent fidèles à l'ancienne manière, concrète et empirique, d'envisager l'exception Giffen. On peut alors leur prêter la définition suivante, d'esprit marshallien: des biens inférieurs (c'est-à-dire dont la demande décroît avec le revenu) et qui absorbent une part importante du revenu. La seconde définition diffère de la première en ce qu'elle fait exclusivement référence à des concepts observables, ou supposés tels, ce qui ne vaut pas pour l'effet de substitution ne l'est pas. Les économistes admettent en effet que les fonctions de demande ordinaire $x_{j}(p, I)$ sont observables, mais non pas celles de demande compensée $h_{j}(p, \bar{u})$. C'est de cette manière quasi-marshallienne que le fondateur de la théorie contemporaine de la demande, Hicks, envisage les biens Giffen (1939-1946, p. 35).

Le choix d'une définition ou de l'autre est important parce qu'il va déterminer la manière de comprendre la formulation contemporaine de la loi de la demande:

"pour tout consommateur, la quantité demandée du bien $\quad j$ diminue lorsque son prix $\quad p_{j}$ augmente, les autres variables restant fixées, si $j$ n'est pas un bien Giffen".

Pour qui retient la définition concrète et empirique des biens Giffen, cet énoncé représente une proposition synthétique, et même, à première vue, une proposition testable. Avec la définition théorique et abstraite, le même énoncé ne représente plus nécessairement une proposition synthétique; tout dépend alors du statut conféré à la maximisation. Supposons que l'on ait pris comme définition du consommateur: "tout individu qui maximise son utilité $u(x)$ sous la contrainte budgétaire $p x=I "$ ". Comme la relation de Slutsky et le signe négatif de l'effet de substitution se déduisent de l'hypothèse de maximisation et d'elle seule, l'énoncé exprime une vérité analytique. Inversement, si l'on rejette la définition précédente du consommateur et que l'on prenne la maximisation comme une proposition synthétique, il exprime à son tour une proposition synthétique.

17 A cause de cette indétermination, la loi de la demande sous sa forme traditionnelle ("pour tout consommateur, la quantité du bien demandée diminue lorsque son prix augmente, les autres variables restant fixées") n'est pas valable inconditionnellement dans la micro-économie contemporaine.

18 Parmi bien d'autres exemples, on lit chez Henderson et Quandt $(1971,2.6)$ qu'un bien sera dit Giffen si "l'effet de revenu est assez important pour contrebalancer l'effet de substitution négatif et rendre $\left(\Delta x_{j}\right)$ positif". 
Les deux définitions sont défendables, quoiqu'elles ne s'accordent pas aux mêmes contextes d'usage. La définition concrète paraît la seule appropriée si l'économiste veut formuler une explication des phénomènes de la demande. La théorie hicksienne explique le cas Giffen par la place que prend l'effet de revenu; mieux, elle réussit à l'expliquer comme exception, puisqu'il faut des circonstances assez particulières pour que l'effet de revenu domine l'effet de substitution. Si on la choisissait dans ce contexte, la définition abstraite conduirait à assimiler directement le phénomène à expliquer et le raisonnement susceptible de l'expliquer. On voit aussitôt qu'elle interdirait de parler véritablement d'explication: ce dernier concept demande une liaison synthétique de ce qu'il relie.

Dans un exposé purement théorique, en revanche, si l'on veut mettre d'abord en évidence le pouvoir unifiant des définitions et des principes, on ne perd rien à traiter la loi de demande comme une proposition analytique. C'est alors sans inconvénient que l'on identifie la notion de bien Giffen et ce qui, précédemment, lui tenait lieu d'explication. Il y a des avantages positifs à procéder ainsi: on évitera que le doute ou la confiance proviennent de raisons extérieures et qu'ils détournent le lecteur des enchaînements qui forment l'objet de l'exposé. Non seulement la première définition des biens Giffen paraît maintenant s'imposer, mais également celle du consommateur par la capacité à optimiser, plutôt que par tel ou tel trait empirique. La micro-économie du consommateur dans son ensemble se présentera comme un tissu de vérités analytiques, la "loi de la demande" n'étant qu'un cas parmi d'autres. 19

Les ouvrages de micro-économie ne précisent pas tous leurs objectifs et, plus couramment encore, ils ne s'en tiennent pas à ceux qu'ils annoncent. Certains multiplient les connotations concrètes à propos du consommateur avant d'opter pour une définition purement abstraite des concepts ultérieurs; d'autres, en sens inverse, font surgir des pseudo-explications de ce qui était présenté d'abord comme un simple jeu de définitions. On pourrait en dire autant de nombreux manuels de physique, où les enjeux de l'exposé n'apparaissent pas distingués comme il conviendrait. Cette comparaison ne vaut pas raison, parce que les physiciens peuvent s'accommoder d'un certain laisser-aller à meilleur droit que les micro-économistes. Pour une discipline où l'identification des lois fait encore problème, il faut mieux pourchasser l'ambiguiité des sources de la vérité, logico-mathématique ou empirique, dans les cas privilégiés où l'on peut en faire la distinction, et la théorie du consommateur est assurément

19 Il faut alors mettre l'expression "loi de la demande" entre guillemets, parce que la notion de loi, comme celle d'explication, exclut l'analyticité. 
l'un de ces cas.

\section{Les biens substituts en micro-économie du consommateur}

Dans la langue ordinaire, "substitut" est synonyme de "remplaçant". Littré dit: "celui qui tient la place ou qui exerce les fonctions d'un autre en cas d'absence ou d'empêchement". Une chose ne se substitue à une autre qu'à un point de vue donné: Littré souligne précisément la fonction commune remplie par la chose et son substitut. Les économistes suivent cette pente lorsqu'ils donnent à penser, et parfois même écrivent, que deux biens substituts répondent aux mêmes besoins. Ce point de vue "objectif" n'est ni le seul possible ni le seul représenté dans la discipline. Beaucoup plus couramment, ils considéreront que deux biens substituts apportent à l'individu les mêmes satisfactions: c'est une autre manière, "subjective" celle-là, d'indiquer leur fonction partagée.

Même si elles se rencontrent dans les ouvrages introductifs, ${ }^{20}$ aucune des remarques précédentes sur les besoins ou la satisfaction ne fournit la définition des biens substituts dont se sert la théorie du consommateur. Depuis l'article de Hicks et Allen (1934) et, surtout, depuis Value and Capital, on définit la substituabilité - ainsi que les notions logiquement associées d'indépendance et de complémentarité - par des propriétés techniques exclusivement fondées sur les concepts de cette théorie. On pose que $j$ est un substitut de $k$ si la demande compensée du bien $j$ varie positivement avec le prix de $k$, ce qui s'écrit avec les notations précédentes:

$(* *) \frac{\partial x_{j}(p, I)}{\partial p_{k}}+\frac{\partial x_{j}(p, I)}{\partial I} \cdot x_{k}(p, I)>0$.

La définition se comprend à la lumière des équations de Slutsky $(*)$ et de la décomposition en deux effets qu'elles autorisent. Comme la substituabilité de $j$ à $k$ n'a rien à voir avec l'effet de revenu indirectement provoqué par la variation du prix $p_{k}$, la théorie élimine purement et simplement cet effet: d'où l'inégalité demandée. On dit que $j$ est complémentaire de $k$ si l'inégalité va dans le sens opposé, et que $j$ est indépendant de $k$ dans le cas d'une égalité à zéro.

20 Comme celui de Henderson et Quandt $(1971,2.6)$. 
L'orientation choisie par la théorie n'allait aucunement de soi. La relation $(* *)$ se rapporte directement aux demandes, c'est-à-dire au comportement du consommateur, alors que la notion ordinaire de substitut privilégie, comme on l'a vu, la raison de ce comportement (la fonction que les deux biens remplissent). On pouvait donc concevoir que les économistes restent plus près du sens commun; ils auraient traité $(* *)$ non pas comme une définition, ce qui en éloigne grandement, mais comme une propriété hypothétique, vérifiée ou non par les substituts compris à la manière naturelle. Des tentatives qui vont dans une direction approchante se sont fait jour aux tout débuts de la micro-économie du consommateur. La définition d'Auspitz et Lieben, que l'on trouve encore dans le Manuel de Pareto (1909), pose que le bien $j$ est substitut de $k$ si:

$\frac{\partial^{2} u}{\partial x_{k} \partial x_{j}}>0$

$u$ dénotant la fonction d'utilité du consommateur. Elle fait référence non pas aux demandes, mais aux variations marginales de la fonction d'utilité - donc, non pas au comportement luimême, mais à ses raisons. La théorie ultérieure se veut ordinale et tournée vers l'observabilité. Elle a rejeté la définition d'Auspitz-Lieben-Pareto parce que les fonctions d'utilité ne sont pas observables et que des transformations ordinales de $u(x)$ peuvent changer le signe des dérivées croisées. ${ }^{21}$ Les micro-économistes d'aujourd'hui ne laissent planer aucun doute sur le fait que la relation (**) leur serve de définition, et cependant, ils perçoivent obscurément que d'autres choix seraient plus naturels. La définition contemporaine est une côte mal taillée. Il en découle une répartition singulière de l'analytique et du synthétique dans cette branche de la micro-économie. Le tableau est assez déconcertant pour qu'on s'y arrête quelque peu.

Soit l'énoncé micro-économique:

"Le bien $j$ est un substitut du bien $k$ si et seulement si $k$ est un substitut de $j$ ".

Représente-t-il une proposition analytique ou synthétique? Si l'on oublie la théorie pour se contenter des prénotions ordinaires, la proposition visée est évidemment analytique. Le sens commun fait référence à une fonction que les biens partagent, ce qui entraîne la conclusion. Peu importe quelle définition explicite on adopte, "subjective" ou "objective", pour peu qu'elle reste conforme au sens commun. L'énoncé précédent résulte alors de la "loi de la logique" voulant toute définition qui prête à $j$ et $k$ uniquement des propriétés qu'ils partagent doive être symétrique relativement à $j$ et $k$. En revanche, la définition explicite de Hicks et les "lois de logique" n'autorisent pas à traiter " $j$ est un substitut de $k$ " comme équivalent à " $k$

21 Pour l'histoire de la théorie de la demande, on consultera Chipman (1976) et Chipman et Lenfant (1999). 
est un substitut de $j "$. Si l'on en restait là, il faudrait conclure au caractère synthétique de la proposition au regard de la théorie, ce qui surprend. ${ }^{22}$

Les micro-économistes n'auraient jamais pris le risque d'introduire une définition qui, en violation patente avec le sens commun, ne serait pas toujours symétrique. Ils supposent en fait acquise une autre proposition fondamentale de la théorie, qui met en relation la demande compensée de $j$ et celle de $k$ :

$(* * *) \frac{\partial x_{j}(p, I)}{\partial p_{k}}+\frac{\partial x_{j}(p, I)}{\partial I} \cdot x_{k}=\frac{\partial x_{k}(p, I)}{\partial p_{j}}+\frac{\partial x_{k}(p, I)}{\partial I} \cdot x_{j}$

C'est la condition bien connue de symétrie des demandes compensées, qui suit de la maximisation de l'utilité, ainsi que Slutsky (1915) l'avait déjà démontré. Elle entraîne la symétrie désirée dans la définition des biens substituts (ou complémentaires, ou indépendants). La définition hicksienne rejoint ainsi le sens commun. ${ }^{23}$

Comme la condition de symétrie de Slutsky découle exclusivement de la maximisation de l'utilité, l'énoncé: "Le bien $j$ est un substitut du bien $k$ si et seulement si $k$ est un substitut de $j "$ exprime une proposition analytique ou synthétique selon l'interprétation choisie pour l'énoncé primordial: "tout consommateur maximise son utilité $u(x)$ sous la contrainte budgétaire $p x=I$ ". Les deux interprétations, l'analytique et la synthétique, coexistent dans la pratique. Chacune présente des avantages et des inconvénients, dont il faut maintenant dresser le bilan.

L'interprétation analytique pour la maximisation individuelle permet de retrouver la symétrie comme propriété analytique, donc universelle, ce qui semble particulièrement appréciable quand on entreprend une classification empirique des biens de consommation en biens substituts, complémentaires et indépendants. Que dirait-on d'un économiste qui rangerait le beurre parmi les substituts de la margarine, mais non la margarine parmi les substituts du beurre? Les travaux appliqués sur la consommation supposent inévitablement que l'on

22 La définition moins élaborée de la substitution de $j$ à $k$, qui repose sur la demande brute ou non compensée: $\frac{\partial x_{j}(p, I)}{\partial p_{k}}>0$

appelle une conclusion identique; cette définition des substituts bruts se rencontre en théorie de l'équilibre général (Arrow et Hahn, 1971).

23 Cette définition prend ici un avantage décisif par rapport à la définition de la note précédente, pour laquelle on ne peut pas démontrer la symétrie. 
regroupe les biens en sous-familles de substituts proches: il n'y aurait guère de sens à effectuer ces découpages si l'on ne tenait pas pour acquise la propriété de symétrie. La résolution par l'analytique présente cependant le défaut considérable, pour garantir une propriété particulière, d'ôter son contenu factuel à la théorie entière. Celle-ci est un enchaînement de déductions suspendues à une prémisse pour ainsi dire unique, la maximisation individuelle. En déclarant analytique la prémisse en question, c'est toute la théorie que l'on se contraint à interpréter de cette façon, et cela, pour sauver une proposition isolée, qui est devenue un enjeu théorique à cause de la définition choisie.

Qu'en est-il maintenant de l'interprétation synthétique pour la maximisation individuelle? Elle évite l'inconvénient précédent. Elle permet d'attribuer le caractère factuel aux implications autres que la symétrie, ce qui est indispensable si l'on veut donner un pouvoir explicatif à la théorie (nous l'avons montré à propos des biens Giffen). En tirant l'interprétation synthétique dans le sens de l'observabilité, on peut même transformer la symétrie en test empirique de la maximisation. Mais ce faisant, on ouvre une boîte de Pandore. Les économistes qui ont testé la symétrie de Slutsky (***) ont obtenu des résultats qui sont, pour dire le moins, mitigés. ${ }^{24}$ Il reste une possibilité: que l'on interprète la maximisation individuelle comme une proposition synthétique tout en déniant le caractère testable à cette conséquence particulière qu'est la symétrie. Il resterait à justifier ce choix d'allure arbitraire alors que d'autres implications directes, comme la négativité de l'effet de substitution propre, feront vraisemblablement l'objet d'une décision différente. De plus, il reste gênant de faire usage de la définition hicksienne sans qu'on sache à quoi s'en tenir pour une exigence aussi élémentaire que la relation symétrique des substituts.

En bref, la distinction de l'analytique et du synthétique se présente comme une véritable alternative pour la définition reçue. Si l'on prend du recul, celle-ci pourra sembler viciée dans le principe: il est singulier que l'on ne puisse la rendre satisfaisante sans remonter d'une façon ou d'une autre à la proposition la plus fondamentale de la théorie! On notera que, malgré tous ses défauts, la définition d'Auspitz-Lieben-Pareto est symétrique, et que, de ce point de vue, elle permet de parler de substituts sans qu'on doive aller chercher plus haut dans la théorie.

On a parfois reproché à la définition hicksienne de biaiser la classification des biens en faveur de la substituabilité. Cette singularité résulte d'une déduction facile, que nous reproduisons

24 Deaton et Mullbauer (1980, p. 79-80) dressent un bilan négatif des tests, et de même, Phlips (1983, ch.3). 
dans l'appendice, parce que les manuels d'aujourd'hui l'omettent généralement. Si l'on somme l'équation obtenue $(* * * *)$ sur tous les biens, on obtient la proposition supplémentaire qu'ils ne peuvent pas être tous mutuellement complémentaires, alors qu'ils peuvent être tous mutuellement substituts. Hicks, qui a lui-même formulé ces résultats, signalait que sa théorie avait pour effet de rendre la substitution de quelque manière plus fréquente que la complémentarité: l'observation perdure discrètement dans la théorie sans qu'on lui ait trouvé de justification. Les manuels choisissent généralement de l'ignorer; il semblerait que nombre d'économistes n'en aient même pas connaissance. On ne la mentionne guère que si l'on veut contester la définition hicksienne des biens substituts et des complémentaires. ${ }^{25}$

Fort des distinctions épistémologiques, nous pouvons situer la critique à un niveau d'abstraction plus élevé que celui du paradoxe intuitif. Si la dissimilarité qui vient d'apparaitre entre substituts et compléments était l'objet d'une proposition analytique, cela voudrait dire, en l'occurrence, qu'elle découle des définitions explicites et des lois de la logique. Il n'est pas concevable que des définitions seules impliquent une fréquence particulière des biens substituts et complémentaires; il faut donc conclure que l'une d'entre elles n'était pas conforme à sa nature de définition. Mais si, d'un autre côté, l'on fait de la fréquence l'objet d'une proposition synthétique, on se heurte à des arguments déjà présentés en liaison avec la symétrie. Considérée comme observable, la proposition synthétique en question ne passerait pas l'épreuve des données; vue comme non testable, elle serait isolée au sein d'un ensemble d'implications que l'on ne veut pas toutes traiter ainsi. La protestation des minoritaires contre la théorie hicksienne apparaît compatible avec les deux branches de la critique épistémologique. Les concepts épistémologiques peuvent les aider à mettre en forme l'insatisfaction qu'ils ressentent. 26

\section{L'analytique et le synthétique en macro-économie. L'équation quantitative de la monnaie}

\footnotetext{
25 Deaton et Muellbauer (1980, p.46) et Phlips (1983, p.79) sont dans ce cas. Malinvaud (1971), comme Hicks, mentionne la propriété sans l'interpréter. Mas-Colell, Whinston et Green (1995, p. 70) sont encore plus allusifs.

26 Sans toucher ni au problème de la symétrie ni à celui de la fréquence, Rosenberg (1976) a déjà discuté l'exemple des biens substituts à l'aide des concepts de ce chapitre. Sa discussion comporte une difficulté: il considère une interprétation synthétique possible pour la relation $(* *)$, que la théorie contemporaine prend exclusivement comme définition des substituts. La position que nous prenons sur l'analytique nous enjoint d'adopter les définitions explicites de l'économiste chaque fois que celles-ci sont disponibles.
} 
Les économistes débattent volontiers de la nature des relations macro-économiques. Leurs interrogations renvoient pour partie au mystère des agrégats, ces quantités représentatives de l'économie dans son ensemble et qui devraient en principe s'obtenir à partir des quantités individuelles correspondantes: le revenu national, la dépense globale, le niveau d'activité, la quantité de monnaie, le niveau général des prix, et ainsi de suite. Mais c'est, plus encore, au moment de relier ces quantités entre elles que les économistes semblent pris de perplexité. Quel sens y a-t-il, par exemple, à poser l'égalité de la dépense et du revenu, de l'épargne et de l'investissement, de la quantité d'argent qui circule avec la masse des transactions? Depuis que l'esprit comptable a fait surgir ces relations, on a pris coutume de leur attribuer deux interprétations possibles: on peut les voir soit comme des identités, utiles pour organiser le raisonnement, mais strictement parlant non informatives, soit comme des équations d'équilibre ou de comportement, ce qui les rend à la fois informatives et contestables. La terminologie qui permet de formuler cette distinction n'est ni satisfaisante ni même unifiée. S'agissant des identités, les économistes disent presque indifféremment "truismes", "évidences", "tautologies", sans parler des tournures aberrantes, comme ce "plutôt tautologique", rather tautological, qui échappe un jour à Hicks à propos de l'équation quantitative de la monnaie. ${ }^{27} \mathrm{La}$ distinction très substantielle qu'indiquent ces termes peut être reprise et clarifiée de plusieurs manières, les unes internes à la théorie économique, les autres proprement épistémologiques. Nous tenterons de la rapprocher de celle, mieux comprise, de l'analytique et du synthétique. Nous avons pris comme exemple l'équation quantitative de la monnaie parce qu'il est célèbre sans être particulièrement difficile à traiter; d'autres opposeraient plus de résistance.

Toutes les variantes de l'équation procèdent de la même formule initiale, qui est l'équation des transactions, $M V=P T$. On porte, à gauche, la quantité de monnaie, $M$, multipliée par la vitesse de circulation de l'unité de monnaie, $V$, et à droite, une mesure physique des transactions effectuées, $T$, multipliée par l'indice des prix, $P$. Naturellement, les prix sont exprimés dans la même unité que $M$ et l'on fixe une unité de temps commune pour mesurer les quatre quantités. C'est la forme de l'équation que retient Fisher dans The Purchasing Power of Money (1911). Voici la justification qu'il en donne:

"Dans chaque vente ou achat, la monnaie et les biens échangés sont ipso facto équivalents; par exemple, la monnaie qui a payé le sucre est équivalente au sucre acheté. Et dans le total de l'ensemble des échanges d'une année, le total de la monnaie payée a une valeur égale à la valeur totale des biens achetés. Les deux membres de

27 Hicks (1935-1967, p. 62). Agassi (1971) relève la bizarrerie. 
notre équation sont donc relatifs l'un à la monnaie, l'autre aux biens. Le membre de la monnaie est le total de la monnaie payée et peut être considéré comme le produit de la monnaie par sa vitesse de circulation. Le membre des biens se compose du produit de la quantité des biens échangés par leurs prix respectifs" (cité par Marchal et Lecaillon, 1967, p. 78).

Fisher considère implicitement que son équation repose sur une identité comptable. Cependant, il s'éloigne de la manière ordinaire de composer un bilan: au lieu de porter simplement, à gauche, la somme des paiements effectués, et à droite, la valeur totale de ce qu'ils ont servi à acheter, il récrit chaque membre comme un produit de quantités totales ( $M$ et $T$ ) par des quantités moyennes (respectivement $V$ et $P$ ). La réécriture vise à introduire des agrégats que l'on peut éventuellement relier par des relations supplémentaires, moins évidentes que l'équation quantitative et qui feront système avec elle. Nous insistons sur l'étape de réécriture parce qu'elle constitue le point de passage de la comptabilité vers l'analyse macro-économique - en l'occurrence, celle que l'on associe, chez Fischer et d'autres, à la théorie quantitative de la monnaie, qu'il faut veiller à distinguer de l'équation du même nom.

Dans ses occurrences post-fishériennes, l'équation quantitative s'écrit généralement $M V=P y$, la quantité $y$ désignant le revenu national à prix constants. Comme le revenu national nominal $Y=P y$ est un concept de valeur ajoutée, la différence avec la formulation de Fisher porte sur la manière de comptabiliser les transactions multiples relativement à un même bien ou service. Alors que $P T$ inclut la valeur de la farine une première fois quand le meunier la vend au boulanger, et une seconde fois quand le boulanger vend le pain au consommateur, $Y$ l'inclut seulement à l'occasion de la première transaction et ne comptabilise la seconde qu'à hauteur de la différence de valeur entre le pain et la farine. En reformulant ainsi le membre de droite, on s'impose de modifier en conséquence le membre de gauche: la quantité $M$ reste ce qu'elle était, mais $V$ représente désormais le nombre de fois qu'une unité de monnaie sert à effectuer un paiement de bien ou de service final. La vitesse de circulation se rapporte donc à une notion hypothétique de transaction. Cette variante offre l'avantage de s'intégrer plus complètement que l'autre à l'analyse macro-économique, puisque le revenu national, nominal ou réel, en est l'une des quantités caractéristiques. Simultanément, elle accroît les difficultés de mesure pour l'agrégat $V$. Ce problème est si sérieux, dit Friedman, que les utilisateurs de l'équation relative au revenu, ont généralement choisi de "calculer $V$ à la valeur qui a la propriété de rendre l'équation correcte" (1974, p.6). S'il en va bien ainsi, il faut conclure que l'équation du revenu ne peut plus passer pour une identité comptable: elle devient une tautologie pure et simple. 
La variante qui est propre à Friedman $(1956,1974)$ éloigne l'équation quantitative à la fois de ses origines comptables et de la réinterprétation tautologisante. Il la retrouve par un détour inattendu, en partant d'une fonction de demande de monnaie:

$$
M^{d}=f\left(P, Y, w, r_{m}, r_{b}, r_{e}, i, u\right)
$$

qui dépend de $P, Y$, de la richesse en termes réels, $w$, des taux de rendements sur différents actifs $r_{m}, r_{b}, r_{e}$, du taux d'inflation $i$ et des facteurs qui affectent les préférences individuelles, résumés dans la variable $u$. Un argument que nous ne détaillons pas amène la conclusion que $M^{d}$ est homogène de degré 1 par rapport aux deux quantités nominales $P$ et $Y$ (en termes plus parlants, Friedman affirme que l'agent est dénué d'illusion monétaire). De la propriété d'homogénéité, il résulte que l'équation de $M^{d}$ peut se récrire:

$\frac{M^{d}}{Y}=f\left(\frac{P}{Y}, 1, w, r_{m}, r_{b}, r_{e}, i, u\right)$

et comme Friedman suppose que la demande de monnaie $M^{d}$ est égale à l'offre fixée $M$, il obtient finalement une variante de l'équation quantitative dans laquelle $V$ est l'inverse de la fonction de demande $f$ :

$M \cdot V\left(y, w, r_{m}, r_{b}, r_{e}, i, u\right)=Y$.

Parce qu'il explicite les variables dans $V$, Friedman est passé d'une identité à une équation, ce que n'étaient pas, malgré leur appellation coutumière, les deux variantes précédemment discutées. Le changement de statut logique s'accompagne d'une transfiguration théorique: la dernière formule écrite est une condition d'équilibre du marché de la monnaie. On peut la comprendre un peu différemment: si l'on omet le côté de l'offre, elle détaille la demande de monnaie en fonction de ses déterminants, c'est-à-dire qu'elle énonce une relation de comportement. 28

En dépit des flottements terminologiques dont ils sont coutumiers, les économistes savent dans l'ensemble distinguer entre l'équation quantitative de la monnaie, comprise d'une façon ou d'une autre, et l'ensemble plus vaste constitué par la théorie quantitative de la monnaie. Sans doute la théorie a-t-elle partie liée, historiquement et logiquement, avec l'équation, mais elle va plus beaucoup loin, puisqu'elle se propose en substance d'effectuer le partage des variations entre les quatre agrégats. Sous sa forme radicale et archaïque, celle de Hume, que l'on retrouve encore chez Fisher, la théorie quantitative affirme qu'une variation de la quantité

28 Friedman n'est ni le seul, ni même le premier, à remplacer les identités comptables par des équations de théorie macro-économique. Les économistes de Cambridge qui l'ont précédé, comme Marshall, Pigou et Keynes, font la transition entre la formulation de Fischer et la sienne. 
de monnaie $\frac{\Delta M}{M}$ induit une variation identique des prix $\frac{\Delta P}{P}$. Il en existe d'autres variantes plus subtiles, comme celle de Friedman justement, mais il n'est pas nécessaire de les développer ici. Nous avons réuni un matériau suffisant pour que l'examen épistémologique puisse prendre le relais.

Les distinctions spontanées effectuent un découpage en trois zones: les tautologies, les identités comptables et les relations macro-économiques proprement dites. Quoi qu'on en prétende parfois, les économistes savent généralement repérer les variantes de l'équation quantitative qui tombent dans la première catégorie, et ils ne leur attachent pas plus d'importance qu'il ne faut. ${ }^{29}$ L'équation quantitative ainsi comprise est un moyen d'organiser la pensée, tout comme le schéma du modus ponens et d'autres tautologies propositionnelles qui servent à encadrer le raisonnement. Les économistes identifient sans hésitation la troisième catégorie, laquelle intervient toujours à l'occasion de variantes nettement spécifiées; la sûreté qu'ils démontrent ici vient de ce qu'ils savent faire la différence entre la théorie et l'équation quantitatives. Philosophiquement, le sort de ces deux catégories est facile à régler: les tautologies sont un cas particulier de propositions analytiques, et les relations d'équilibre ou de comportement peuvent s'interpréter comme analytiques ou synthétiques suivant les stéréotypes dominants de l'activité scientifique poursuivie.

L'incertitude éventuelle se concentre sur la catégorie intermédiaire des identités comptables. Or les économistes ne l'appréhendent pas bien: ils sont inutilement dépréciatifs à son égard et ne la subdivisent pas comme ils devraient. Pour le montrer, revenons au tout premier énoncé: la somme des paiements est égale à la somme des achats réalisés sur la même période. On peut considérer que la proposition correspondante ne fait que répéter la définition des mots "paiement" et "achat"; elle est alors analytique en un sens quasiment frégéen; elle n'est certainement pas une tautologie. Adoptant un point de vue très différent, on peut vouloir considérer que, dans l'énoncé précédent, les mots "paiement" et "achat" ne prennent pas leur sens coutumier, qu'ils désignent des mesures d'un certain genre effectuées par l'institut de statistique, et que ces mesures qu'ils désignent constituent en fait leurs significations. Il faut alors se demander si, oui ou non, les paiements et les achats font l'objet de mesures indépendantes; dans le premier cas, la proposition devient synthétique; dans le second, elle reste analytique, quoique ce soit en un sens qui ne dépend plus des définitions explicites. Il est

29 Hutchison (1938) fait à cet égard un bien mauvais procès à la théorie économique; nous discutons sa thèse dans la section suivante. 
remarquable qu'une double possibilité d'interprétation apparaisse pour l'énoncé comptable le plus sommaire de tous. Nous venons de mettre en évidence une généralité: les propositions comptables ne sont pas intrinsèquement analytiques ou synthétiques.

Soit l'énoncé comptable qui suit, $M V=P T$. Il faut à nouveau se demander si les mots, ou dans ce cas, les symboles, ont le sens coutumier, auquel cas la proposition correspondante est analytique, ou s'ils n'auraient pas plutôt un sens qui dépende des procédés de mesure employés. Voici une configuration qui, parmi d'autres, rendrait la proposition synthétique: $M$ et $P$ sont mesurés indépendamment; la somme des paiements annuels et celle des achats annuels ont la même mesure; on obtient $V$ en divisant la somme des paiements par $M$; mais on dispose pour $T$ d'une mesure indépendante. Admettons maintenant que l'on obtienne la valeur de $T$ en divisant la somme des rentrées par $P$; alors, la proposition retombe dans l'analytique.

On peut reprocher à la catégorie d'identité comptable, telle que les économistes la manipulent, d'obscurcir la différence pourtant économiquement significative de ces différents cas. Par contraste, on la fait apparaître dès qu'on soulève la question - apparemment artificielle et extérieure - de l'analytique et du synthétique. On est alors forcé de rechercher, s'il s'en trouve, les définitions explicites, et sinon, de préciser les significations sous-jacentes. Les économistes qui suggèrent, sans le dire en ces termes, que les identités comptables sont par nature analytiques, se limitent en fait à une configuration particulière des mesures. Le paragraphe précédent l'a décrite en dernier lieu: la masse monétaire $M$ et l'indice des prix $P$ sont mesurés indépendamment par l'institut de statistique, les sommes des deux membres ont la même mesure, les deux notions restantes, $V$ et $T$, sont obtenues par division. La mauvaise réputation de l'équation $M V=P T$ provient de cette configuration, qui n'a pourtant rien d'intrinsèque. Elle est aggravée par la possibilité qui s'ouvre de mesurer $M$ de différentes façons. 30

Des malentendus sont apparus lorsque les économistes ont fait mine de tester non pas seulement les propositions spéciales de la théorie quantitative, ce qui était légitime et naturel, mais l'équation quantitative elle-même. Il va de soi qu'on ne teste pas les propositions analytiques. Mais dans le cas d'un énoncé comptable, on peut procéder à des vérifications en un sens plus modeste. L'actif d'un bilan doit être égal à son passif: c'est ce que dit l'énoncé

\footnotetext{
30 En général, on lit l'équation des transactions en prenant pour $M$ le stock total de monnaie, et non pas la quantité de monnaie qui a circulé pendant la période (Patinkin, 1969, Friedman, 1974). Il reste ensuite à décider quel genre de monnaie bancaire on inclut dans $M$ au-delà de la monnaie fiduciaire.
} 
comptable, analytiquement interprété. Le bilan peut être faux: c'est ce qui dit le même énoncé, synthétiquement interprété. Quand les mesures des concepts sont indépendantes, il est coutumier de rapprocher les deux sommes de l'actif et du passif. Si elles diffèrent, on conclut soit que l'on s'est trompé en prenant ses mesures, soit que les procédés de mesure eux-mêmes étaient défectueux. Les économistes auxquels on reproche de "tester" l'équation quantitative nous paraissent l'avoir en fait vérifiée à la manière banale des comptables: ils se sont assurés par exemple qu'ils ne comparaient pas les "paiements" et les "achats" sur deux périodes différentes, que la mesure de $V$ s'accordait avec celle de $M$, que leurs agrégats renvoyaient à des stocks ou à des flux de manière cohérente (ce dernier point a fait l'objet de querelles chez les quantitativistes). Comme d'autres identités du même genre, l'équation quantitative a rendu des services appréciables à la macro-économie parce qu'elle est une identité comptable, et non pas en dépit de ce trait. Nous insistons sur ce point parce que la tendance, aujourd'hui, est à lire "vérifier" au sens exclusivement du test, ce qui entretient une image de stupidité l'économiste cherchant à confirmer ce qu'il saurait déjà - alors qu'il se conforme simplement au bon sens. 31

\section{Confusions dans le métadiscours}

Cette section déporte l'examen de l'analytique vers l'emploi que les économistes font euxmêmes de ce concept. Il est toujours facile de pointer du laisser-aller chez ceux dont l'exactitude philosophique ne représentait pas l'objectif principal, et il y aurait une supériorité mal comprise à pousser la critique trop loin dans cette direction. Mais il arrive que certaines confusions menacent de descendre de l'étage du commentaire, où elles ont peu d'importance, à celui de la démarche positive, qu'elles risquent alors d'embrouiller inutilement. Les deux exemples qui suivent sont de cet ordre.

Beaucoup parlent de tautologies pour désigner, en fait, l'analytique en général. L'économiste autrichien von Mises $(1933,1949)$ avait coutume d'écrire que les propositions fondamentales de l'économie seraient des "tautologies", et il illustrait sa thèse par l'exemple suivant: "Toute action humaine est rationnelle". On peut à la rigueur défendre une interprétation analytique pour la proposition qu'exprime cet énoncé: il n'est pas absurde à première vue de le mettre en parallèle avec "Tout corps est étendu". Mais il ne peut être un seul instant question d'en faire

\footnotetext{
31 Agassi (1971, p. 55) écrit: "l'équation (quantitative) est un truisme. Cependant, elle était manifestement vue comme empirique plutôt que tautologique: elle a été contestée vigoureusement et personne ne conteste une tautologie en la sachant". Il nous semble exagérer l'état de confusion dans lequel il trouve la théorie.
} 
une vérité logique, sur le même pied que "Tout corps est un corps". Plus correctement formulée, la thèse de von Mises veut donc que les propositions fondamentales de l'économie constituent des vérités analytiques. ${ }^{32}$ Dans son esprit, l'affirmation visait à relever le statut de ces propositions, en faisant ressortir leur évidence intrinsèque et leur caractère indiscutable. Le choix de la terminologie rend incompréhensible l'intention valorisante qui était pourtant celle de l'auteur.

Le britannique Hutchison (1938) acceptait et même radicalisait l'affirmation de l'autrichien la théorie économique contiendrait seulement des "tautologies" - mais il enchaînait différemment: pour cette raison même, elle serait promise à la stérilité. ${ }^{33}$ Parmi ses exemples privilégiés se trouve justement la théorie quantitative de la monnaie. Il est très frappant de voir qu'à partir d'un diagnostic identique, la valorisation puisse partir dans un sens opposé. C'est Hutchison, et non pas von Mises, qui est cohérent avec l'usage dépréciatif du mot "tautologie". Mais sa position globale est radicalement incorrecte, alors que celle de von Mises mérite encore d'être discutée, une fois qu'on a pris la peine de la reformuler. En effet, la thèse de Hutchison contre la théorie économique a besoin non pas seulement du mot, mais de l'idée même de tautologie: c'est la vacuité qu'il veut en effet lui reprocher. Or cette propriété désavantageuse qu'il peut faire valoir à propos des tautologies ne s'applique absolument pas aux propositions analytiques en général. Le diagnostic de Hutchison n'a finalement d'autre motif que l'amalgame des deux classes de propositions. Pour revenir son exemple privilégié, il dénonce la théorie quantitative à partir de l'équation, tout en ignorant les différentes versions possibles de l'équation (toutes, nous l'avons montré, ne peuvent être dites tautologiques, et elles ne sont pas même toutes analytiques). L'objection fondamentale que Hutchison avançait contre la théorie quantitative et, plus généralement, contre ce qu'il appelle "propositions de théorie pure", frappe rétrospectivement comme étant nulle et non avenue.

Von Mises et Hutchison avaient l'excuse d'écrire pendant l'entre-deux-guerres, à une époque où les philosophes eux-mêmes ne disposaient pas encore de toutes les distinctions convenables. ${ }^{34}$ L'amalgame de l'analytique avec les tautologies a malheureusement perduré

\footnotetext{
32 Ce qu'il écrivait aussi du reste: "Le raisonnement aprioriste est purement conceptuel et déductif. Il ne peut rien produire d'autre que des tautologies et des jugements analytiques" (1949, p.38).

33 "On ne voyait pas quelle autre forme de propositions que les "tautologies pures et simples" (mere tautologies) on cherchait à obtenir par la théorie pure" (1938, p. 28).

34 Contrairement à ce que fera Quine, Wittgenstein et Schlick ne distinguaient pas les tautologies des autres propositions analytiques. La thèse néo-positiviste fameuse contre l'a priori se formulait alors comme une thèse de réduction de l'a priori aux "tautologies", et non pas à l'analytique.
} 
après le moment où les philosophes eurent effectués les clarifications nécessaires. En poussant l'enquête, on découvrirait que le mot "tautologique" sert encore aujourd'hui dans les échanges de la discipline comme il servait autrefois à von Mises et Hutchison: tantôt pour élever, tantôt pour rabaisser le statut d'une proposition, et dans tout les cas, comme un point d'arrêt à la discussion. C'est en cela que réside finalement la conséquence la plus regrettable: on s'interdit de discuter de propositions qui devraient être examinées plus avant. Car les propositions analytiques peuvent être critiquées ou défendues en raison des définitions adoptées ou des significations retenues; elles renvoient l'économiste à sa liberté de décision; mais il n'y a rien à dire pour ou contre les tautologies.

\section{Relevé de conclusions}

En amplifiant les leçons des exemples, on retiendra finalement quelques traits saillants de la distinction de l'analytique et du synthétique telle qu'elle se présente en économie. En premier lieu, il est apparu que la distinction dépendait du contexte d'une manière justifiable et souvent - quoique non pas toujours - comprise et dominée par les praticiens eux-mêmes. De nombreux énoncés - comme la définition des substituts en micro-économie du consommateur - ont une interprétation fixe et reconnue dans la théorie économique contemporaine. Certaines notions - comme celle des biens Giffen - peuvent sans doute recevoir des définitions variables, mais alors, le contexte permet d'en sélectionner une aux dépens des autres. Quand les praticiens jugent confusément de ce qu'ils font, nous avons pu apporter des éclaircissements sans nous éloigner trop de ce qu'ils disaient; il en allait ainsi dans le cas des identités comptables. Du moins pour les exemples discutés ici, il n'a pas été nécessaire de solliciter le contexte externe; nous n'en ferons pas une généralité, mais il ressort du moins de ces exemples qu'il n'est pas toujours requis d'examiner les circonstances de l'énonciation lorsqu'on veut interpréter convenablement les énoncés. Quels que soient les rôles respectifs des deux contextes, le point important est qu'ils jouent sur les interprétations de manière suffisamment réglée pour que la distinction de l'analytique et du synthétique échappe à l'arbitraire même si elle varie. ${ }^{35}$

En second lieu, le choix d'une interprétation pour un énoncé donné peut imposer l'interprétation analytique ou synthétique d'autres énoncés, à distance de celui-ci: nous l'avons démontré à propos de la condition de symétrie de Slutsky. Il se confirme ainsi que la

35 Il est finalement curieux que le holisme de Quine, surtout quand il en restreint l'amplitude après 1951, ainsi que son pragmatisme affiché, ne l'aient pas orienté vers une considération de ce genre. 
distinction ne s'appréhende qu'à l'échelle des énoncés pris en groupe. En l'occurrence, il faut souvent remonter au niveau d'une théorie entière, comme celle du consommateur, pour l'appliquer correctement. Il n'y a pas, et il ne peut pas y avoir, de règle pour déterminer la taille de l'unité d'appréciation, mais les différents choix peuvent du moins s'ordonner le long d'une échelle préalable, qui est fixée par les découpages théoriques préexistants.

En troisième lieu - et cette observation, contrairement aux précédentes, est plus particulière à l'économie - on ne doit pas attendre que la distinction se présente à l'identique d'après le langage ordinaire et d'après le langage spécialisé, alors même que le second emprunte les mots du premier. Un décalage excessif avec le langage ordinaire compromettrait non seulement l'utilisation pratique, mais le développement interne de la théorie économique. Les économistes s'efforcent donc de limiter les écarts, ainsi que nous l'avons montré pour le mot "substitut". Mais les choix impliqués pour résorber le décalage d'un côté peuvent l'aggraver d'un autre, et ce dilemme, qui perturbe la micro-économie du consommateur aujourd'hui encore, ramène la question précédente, celle de l'unité convenable d'appréciation.

En quatrième lieu, la distinction permet de comprendre mieux le travail poursuivi en économie et nous pensons même qu'elle puisse quelquefois rendre service à l'économiste luimême. Bien maîtrisée, elle évitera de chercher les preuves de relations admises en vertu des significations ou, symétriquement, d'omettre les preuves de propositions qui en demanderaient. Dans la terminologie qui leur sied, beaucoup pratiquent déjà la distinction de cette manière même; il s'agit alors seulement de leur apporter un surcroît de précision. Même si quelques-uns s'embrouillent et si les manuels comportent un lot excessif d'approximations, la théorie économique ne nous est pas apparue, au regard de l'analytique et du synthétique, dans l'état de confusion que décrivent certains de ses observateurs. La confusion réside le plus souvent du côté de ceux qui la dénoncent. C'est un fait de la discipline dont il serait possible de donner bien d'autres preuves: le métadiscours sur l'économie (la "méthodologie") est plus souvent inadéquat que la pratique elle-même.

\section{BIBLIOGRAPHIE}

Agassi, J. (1971), "Tautology and Testability in Economics", Philosophy of the Social Sciences, 1, p. 49-63. Arrow, K.J. et F.H. Hahn (1971), General Competitive Analysis, San Francisco, Holden Day. 
Carnap, R. (1936-1937), "Testability and Meaning", Philosophy of Science, 3 (1936), et 4 (1937). Reproduit dans H. Feigl et M. Brodbeck, Readings in the Philosophy of Science, New York 1953, p. 47-92.

Carnap, R. (1947), Meaning and Necessity, Chicago, University of Chicago Press (2 2 ème éd. révisée, 1956).

Chipman, J.S. (1976), "The Paretian Heritage", Cahiers Vilfredo Pareto. Revue européenne de sciences sociales, 14, p. 64-173.

Chipman, J.S. et J.S. Lenfant (2002), "Slutsky's 1915 Article : How It Came to be Found and Interpreted", History of Political Economy, 34, p. 553- 597.

Deaton, A. et D. Muellbauer (1980), Economics and Consumer Behavior, Cambridge, Cambridge University Press.

Duhem, P. (1906), La théorie physique, son objet, sa structure, Paris; $2^{\text {ème } e ́ d . ~ a u g m e n t e ́ e, ~ P a r i s, ~ 1914, ~ r e ́ e ́ d . ~ V r i n, ~}$ Paris, 1981.

Fisher, I. (1911), The Purchasing Power of Money, New York, MacMillan (2 $2^{\text {ème }}$ éd. révisée, 1922).

Frege, G. (1884), Grundlagen der Arithmetik, Breslau, Marcus. Trad. fr. par C. Imbert, Les fondements de l'arithmétique, Paris, Le Seuil, 1969*.

Friedman, M. (1956), "The Quantity Theory of Money - A Restatement", in M. Friedman (sous la dir. de) Studies in the Quantity Theory of Money, Chicago, University of Chicago Press, p. 3-21.

Friedman, M. (1970), "A Theoretical Framework for Monetary Analysis", Journal of Political Economy, 78, p.193-238. Repris dans R.J. Gordon (1974) (sous la dir. de), Milton Friedman's Monetary Framework, Chicago, Chicago University Press, p. 1-62.

Gillies, D. (1993), Philosophy of Science in the Twentieth Century, Oxford, Blackwell.

Gochet, P. (1978), Quine en perspective, Paris, Flammarion.

Grayling, A.C. (1997), Introduction to Philosophical Logic, Oxford, Blackwell (3 ${ }^{\text {ème }}$ éd.)

Grice, H.P. et Strawson, P.F. (1956), "In Defense of a Dogma", Philosophical Review, 65, p. 141-158.

Haack, S. (1978), Philosophy of Logic, Cambridge, Cambridge University Press.

Henderson, J.M. et R.E. Quandt (1971), Microeconomic Theory, New York, McGraw-Hill. Trad. fr. Microéconomie. Formulation mathématique élémentaire, Paris, Dunod, 1972.

Hicks, J.R. (1935), " A Suggestion for Simplifying the Theory of Money", Economica, repris dans Critical Essays in Monetary Theory, Oxford, Clarendon Press, 1967, ch. 4, p. 61-82.

Hicks, J.R. (1939), Value and Capital, Oxford, Clarendon Press; 2ème éd. 1946 (les références sont à cette édition).

Hicks, J.R. et Allen (1934), "A Reconsideration of the Theory of Value, I, II", Economica, 14, p. 52-76 et 196219.

Hintikka, J. (1973), Logic, Language-Games and Information, Oxford, Clarendon.

Hutchison, T. W. (1938), On the Significance and Basic Postulates of Economics. Rééd. avec une nouvelle préface, New York, A.M. Kelly, 1960.

Kant, E. (1781), Kritik der reinen Vernunft. Trad. fr. par A. Tremesaygues et B. Pacaud, Critique de la raison pure, Paris, Presses Universitaires de France, 1971.

Katz, J.J. (1990), "The Refutation of Indeterminacy", Journal of Philosophy, 85, p. 227-252. 
Malinvaud, E. (1971), Leçons de théorie microéconomique, Paris, Dunod.

Marchal, J. et J. Lecaillon (1967), Les flux monétaires, Paris, Cujas.

Marshall, A. (1890), Principles of Economics, Londres, MacMillan ( $8^{\text {ème } e ́ d . ~ m o d i f i e ́ e, ~ 1920, ~ r e ́ i m p r . ~ 1949) . ~}$

Mas-Colell, A., M. Whinston et J.R. Green (1995), Microeconomic Theory, Oxford, Oxford University Press.

von Mises, L. (1933), Grundprobleme der Nationalökonomie, Iena, Gustav Fischer (trad. anglaise, Epistemological Problems of Economics, New York, New York University Press, 1976).

von Mises, L. (1949), Human Action. A Treatise on Economics, New Haven, Yale University Press.

Patinkin, D. (1969), "The Chicago Tradition, the Quantity Theory, and Friedman", Journal of Money, Credit, and Banking, 1, p. 46-70.

Phlips, L. (1983), Applied Consumption Analysis, Amsterdam, North Holland.

Proust, J. (1986), Questions de forme, Paris, Fayard.

Putnam, H. (1975), Mind, Language and Reality. Philosophical Papers, 2, Cambridge, Cambridge University Press.

Quine (van Orman), W. (1951) From a Logical point of View, Cambridge, Mass., Harvard University Press; $2^{\text {ème }}$ éd. révisée, 1961; $3^{\text {ème }}$ éd. avec une nouvelle préface, 1980.

Rosenberg, A. (1976), Microeconomic Laws, Pittsburgh, University of Pittsburgh Press.

Slutsky, E. (1915) "Sulla teoria del bilancio del consumatore", Giornale degli economisti e rivista di statistica, 51, p.1-26. Trad. anglaise dans G.J. Stigler et K. E. Boulding, Readings in Price Theory, Homewood (Ill.), Irwin, 1952, ch. 2, p. 27-56.

\section{APPENDICE}

La maximisation de l'utilité sous la contrainte linéaire $p x=I$ implique la condition d'homogénéité de degré 0 des fonctions de demande, condition que l'on peut récrire sous une forme différentielle commode: pour tous les biens $j$ et $k$,

$\frac{\partial x_{j}}{\partial I} I+\sum_{k} p_{k} \frac{\partial x_{j}}{\partial p_{k}}=0$.

En utilisant $I=\sum_{k} p_{k} x_{k}$, il vient:

$\sum_{k} p_{k}\left(\frac{\partial x_{j}}{\partial p_{k}}+\frac{\partial x_{j}}{\partial I} x_{k}\right)=0$

ce qui interdit au bien $j$ d'avoir uniquement des compléments, alors qu'il peut avoir uniquement des substituts puisque, suivant la théorie, l'effet de substitution propre est négatif:

$\frac{\partial x_{j}}{\partial p_{j}}+\frac{\partial x_{j}}{\partial I} x_{j}=\frac{\partial h_{j}(p, \bar{u}(p, I))}{\partial p_{j}}<0$. 
